Vol. 2, No. 2 | July - December 2019

\title{
A High State of Modular Transistor Design on A 105 Kw HVPS for X-Rays Tomography Applications
}

\author{
Hamayun Khan ${ }^{1,4}$, Muhammad Yousaf Ali Khan ${ }^{1}$, Qaisar Bashir ${ }^{2}$ Amad ud din ${ }^{3}$, \\ Kashif Janjua ${ }^{4}$,Shahid Khan ${ }^{5}$
}

\begin{abstract}
:
X-rays tomography work for the production of enhanced imaging at low radiation rate. This technique uses two tube of high voltage input power supply (HVPS) at different values, but such type of technique is so expensive. Another technique that have single tube of high voltage power supply have the ability to produce same high voltage at high rate of switching. This technique is so fast and less costly than the previous technique. In this paper, such type of high voltage input power supply (HVPS) technique is used. This technique consists of high state of modular transistor known is IGBT. The IGBT converts the applied power supply into high voltage power as input supply (HVPS) at the rate of $71 \mathrm{kHz}$, due to which the efficiency is increases to work for high values of load. This technique is tested for both resistive load and X-ray tube and their results are presented. This developed technique is also usable for other applications which are operated on high voltage power supply (HVPS). 3
\end{abstract}

Keywords: X-ray, high voltage power supply (HVPS)

\section{Introduction}

$\mathrm{X}$-ray tomography works to radiate rays to obtain images of an object at different level of applied voltage. As we know x- rays have the ability to have different attenuation at the different level of energy. As a result, the images have more information about every parts of the object as compared to mano energy scan techniques. To replace dual scanning of an object the CT scanners were introduces of dual source. Now days this technology is so entrenched [1]. This technique consists of high voltage two tube for input power supply. One tube has high voltage typically $140 \mathrm{kV}$ and the other have low voltage typically $80 \mathrm{Kv}$, but unfortunately this technique is so costly another technique we want to introduce that have single tube of high voltage power supply having the ability to produce the same voltages as in the previous technique at high switching to change from voltage level to another, as result we can use multi-level of energy with one single tube.

${ }^{1}$ Department of Electrical Engineering, Gomal University, D.I.Khan, KPK, Pakistan

2 Department of Electrical Engineering, University of Lahore, Lahore Punjab, Pakistan

${ }^{3}$ Department of Physics, Fatima Jinnah Women University, Rawalpindi, Pakistan

${ }^{4}$ Department of Electrical Engineering Superior University, Lahore, Punjab, Pakistan

${ }^{5}$ United Consulting Services, D.I.Khan, KPK,Pakistan SJET | P-ISSN: 2616-7069 | E-ISSN: 2617-3115 | Vol. 2 | No. 2 | ( 2019 Sukkur IBA 
There are some basic ideas, e.g., in [2, 3]. The modulation in the range frequency will be 10 $100 \mathrm{~Hz}$ depend on the uses and our need the switching from one level of energy to another is so fast typically less than 100-200 $\mu$ s that generate quasi-rectangular pulses such type of technique gives excellent performance at low cost such technique in case of DC is known as a long-pulse HVPS. Generally, there are some devices which increase the size of the charging power but decrease the output efficiency. We need to overcome these types of challenges [4].

When the frequency is increased without the use of converter module as a result efficiency is going to decrease. In this paper we have to abolish this type of weakness and bring the developmental work for CT \& DE. Heat control by using separate hardware architecture and bit-partitioning method is illustrated in [5]. The author considered an extra efficient mechanism by inducing newer memory chips for accessing register files. Bitpartitioned Register File (BPRF) from basic cache organization mechanism is considered. It is designed on a conventional dynamically scheduled superscalar processor $[6,7]$.

\section{Main design parameters}

High voltage power supply CT scan have major application in the field of medical which have maximum power of about 105 $\mathrm{kW}$. Our major target is to bring down the size and also the cost. We have to make HVPS so strong that it can support the rotational force of typically 50 G. HVPS have two tube for power supply and also high-speed rotor for the rotation of anode.

The specifications are shown in Table 1, the expository part of the high voltage power supply technology is focused conversion from DC to DC. The HVPS has the flexibility and compatibility applications which are the main features.

\section{HVPS design}

The simplified block diagram is shown in below figure 1 . The small size and low-cost capacitive filter are used in the AC chassis. The chassis filter, the large component of RL is at the HV side. An inductive adder is used for the conversion of DC to DC which consists of two module M1 and M2 which phase shifted the HF ripple suppression [8]. While satisfying the specification of HF ripple with the help of two circuit known as voltage multiplicative R1, R2. The high frequency switching circuit has low capacity typically of less than $200 \mathrm{pF}$. Due to this the converter is so nimble and useful for fast transition of low voltage state to high voltage state. As we know there are two inverter cells M1 and M2 as shown in below figure consisting of halfbridge inverters INV1, INV2. The zerocurrent switching (ZCS) mode, the inverter is operated in low line while at full load the inverter operates at $70 \mathrm{kHz}$. In the other mode, the low frequency is observed. The IGBT is used which has less loss and low cost $[11,12]$.

Table I: Descriptions of HVPS for DE applications.

\begin{tabular}{|l|l|}
\hline Peak output power & $105 \mathrm{~kW}$ \\
\hline Average power & $6 \mathrm{~kW}$ \\
\hline Load voltage & $-65 \mathrm{kV}$ to $-145 \mathrm{kV}$ \\
\hline Load voltage regulation & $\pm 0.15 \%$ \\
\hline High frequency (HF) ripple & $<4 \%$ peak-to-peak \\
\hline Low frequency (LF) ripple & $<0.4 \% \mathrm{rms}$ \\
\hline Output Stored Energy & $<6 \mathrm{~J}$ \\
\hline Load current & $12 \mathrm{~mA}$ to $930 \mathrm{~mA}$ \\
\hline Conversion frequency & up to $60 \mathrm{kHz} ;$ \\
\hline Input Voltage & 3 phase $400 \mathrm{~V} \mathrm{AC}$ \\
\hline Typical cable capacitance & $200 \mathrm{pF}$ \\
\hline Dynamic Response: & slew rate $>1 \mathrm{kV} / \mathrm{us} ;$ \\
\hline DE pulse repetition rate (PRR) & $10-1500 \mathrm{~Hz}$ \\
\hline Depth of Modulation (DoM) & zero to $100 \%$ \\
\hline Insulation (HV unit) & Solid \\
\hline Lifetime & $\begin{array}{l}5 \times 10^{\circ} \text { shots at } 50 \% \text { DoM and } 10^{7} \\
\text { shots at } 100 \% \mathrm{DoM}\end{array}$ \\
\hline
\end{tabular}




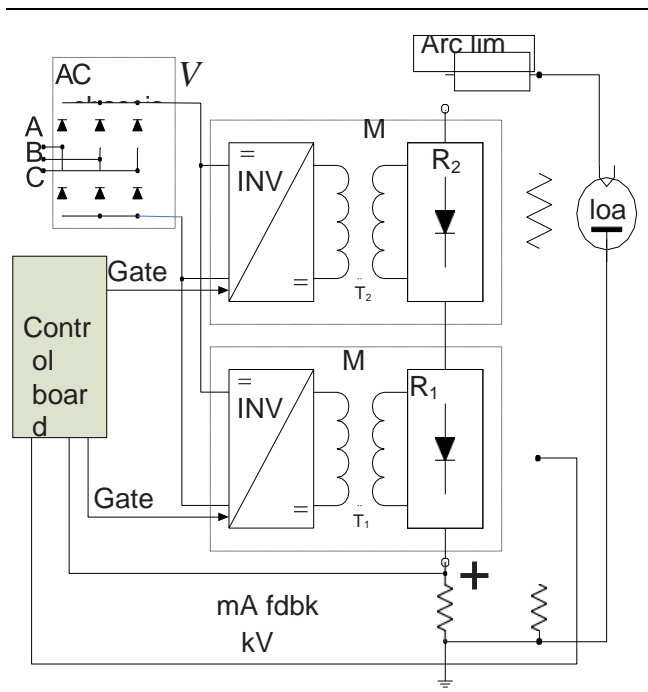

Fig 1: Block-diagram of HVPS.

The two transformers $\mathrm{T} 1$ and $\mathrm{T} 2$ are operated by eight inverters and each transformer have four inverters which are enabled and disabled according to the current operational condition. As the HV transformers have leakage inductance which consists of resonant tank circuits so there is no need of external inductors. The inverter consists of two chassis known as inverter chassis as shown in figure 2 and high voltage chassis as shown in figure 3. High voltage chassis provide insulation for hardness while inverter chassis have importance for the high rotational force of typically 50G. A lot of stress is observed on insulation and other parts during the pulse mood operation. We should need specific insulation system at DE pulse mode. The insulation design is best after testing obliges by FEA.

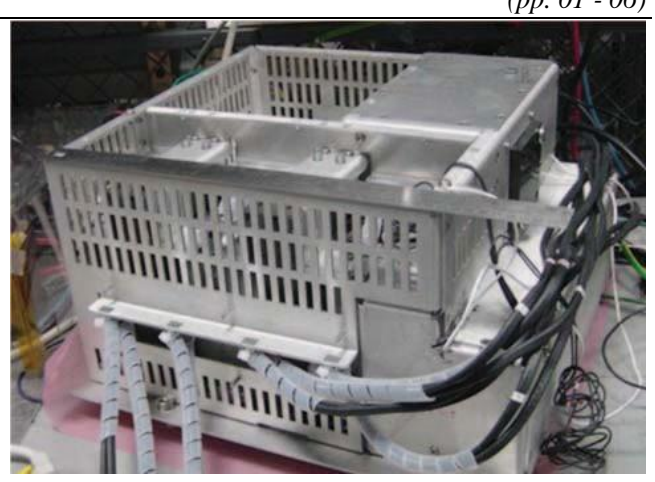

Fig 2: Inverter chassis having Weight of $37 \mathrm{~kg}$.

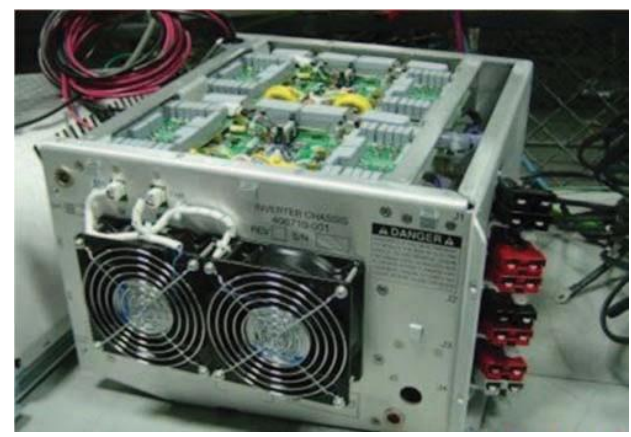

Fig 3: High voltage chassis consist of two transformers having secondary of $140 \mathrm{kV}$.

The base of control section is FPGA which has standard features and so fast digital processing which consist of multiple protection like arc/spark, overheating of major component, over or under current and voltage and the interlock through current and voltage programing. In the frequency mode the inverters are active to regulate the output. When the load voltage in switching frequency increases for every period Ts, the PID control are not able to obtain the flat-top pulses in the wide range of input lines and loads which is changeable task in the case when line and load are fixed.

When the load voltage raises the inverter operates at Maximum frequency and as result 
maximum power charging is obtained and reaches to the desired level. At this stage the converter is in feedback control and frequency is derived by this equation.

$$
\begin{aligned}
& P_{L}=4 f_{s} C_{r} V_{r}^{2} \\
& \text { where } \\
& P_{L}=\text { Load Power } \\
& f_{s}=\text { Switching frequency } \\
& C_{r}=\text { Resonant frequency } \\
& V_{r}=\text { DC rail voltage } \\
& f_{c t}=\frac{P_{L}}{2 * 2 C_{r} V_{r}^{2}}
\end{aligned}
$$

We observed that PSpice model of HVPS is at full scale. All the features of HVPS are reproduced which are phase shifted in addition to the feedback loop.

\section{Experimental results}

Many HVPS are manufactured on compliance for specification. The wave form is obtained for resistive load and X-ray load are given below. Figure 4 is the wave form that is obtained for resistive load with high voltage switching in the range of $-80 \mathrm{kV}$ to $-135 \mathrm{kV}$. The rise time is 100us at which power is high typically at $105 \mathrm{kw}$ for low load and decreases at high load. Figure 5a shows low line collector current at full power.

The Zero Current Switching (ZCS) remains the same and switching frequency reach to 70 $\mathrm{kHz}$. At lower load and high load, the (ZCS) is also observed. Primary current which is phase shifted of the transformer T1, T2 having two inverters inside each module M1 and M2. At high load the collector current is indicated as shown in figure $5 \mathrm{~b}$. Due to non-ideal decoupling in each module some asymmetry is produce in the primary current.
When the test is started for the $\mathrm{x}$-ray tube there should be measurable device to measure the external applied voltage so that we will be able to provide the needed voltage to the $\mathrm{x}$ - ray tube. If the external applied voltage is less than the needed voltage, then the feedback divider is used to increase the external applied voltage.

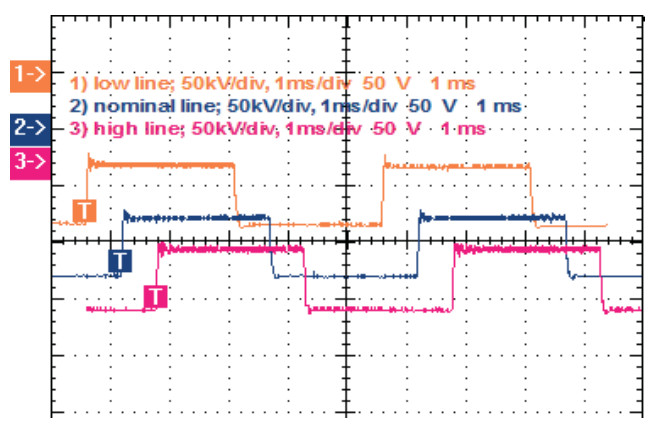

Fig 4: Resistive load $=181 \mathrm{kT}$.

High voltage $=-135 \mathrm{kV}$

Low voltage $=-80 \mathrm{kV}$

$\mathrm{PRR}=200 \mathrm{~Hz}$.

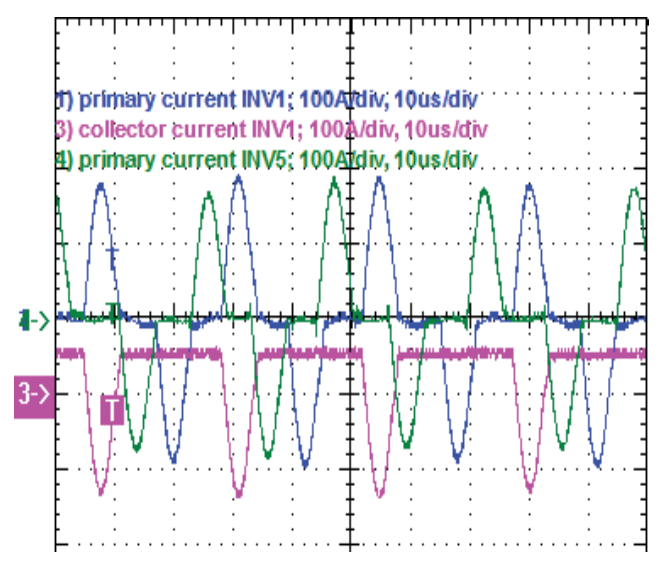

Fig 5a: low line collector current and full power. 


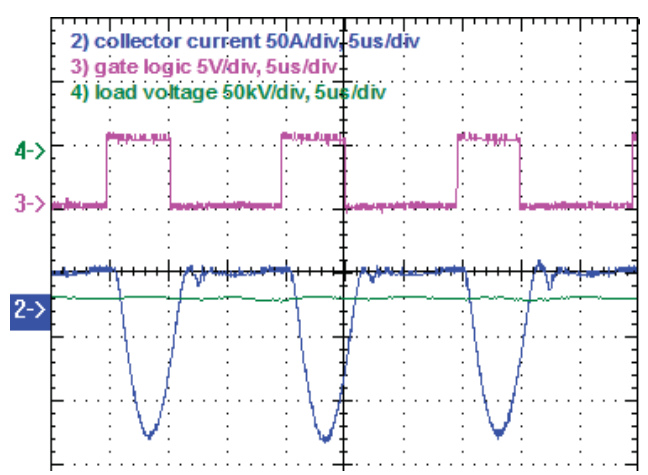

Fig 5b: Collector current due to non-ideal decoupling.

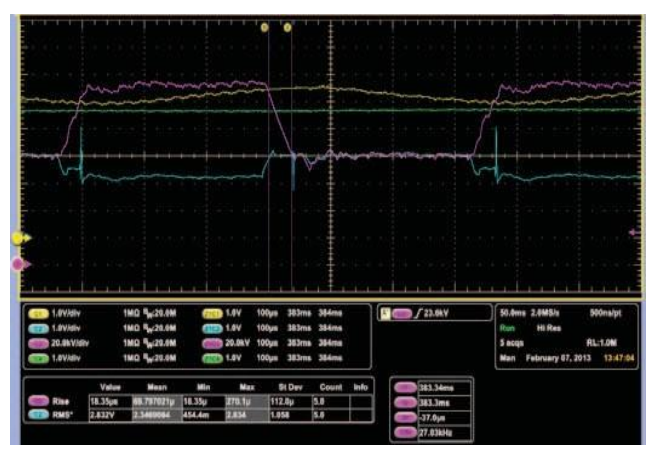

Fig 6: X-ray tube.

High voltage $=-135 \mathrm{kV}$

Low voltage $=-80 \mathrm{Kv}$

Load current $=533 \mathrm{~mA}$

$\mathrm{PPR}=200 \mathrm{~Hz}$

To operate the x-ray tube efficiently, the maximum error is $1.5 \%$ due to the changing of the applied voltage after 200us. The wave form obtained during this operation are given in figure 6 , and figure 7 . When the frequency of the wave is increased from $1 \mathrm{kHz}$ due to the rise and fall so that it takes another $100 \mathrm{usec}$ to settle its efficient value. So, the average frequency for the implementation of this operation.

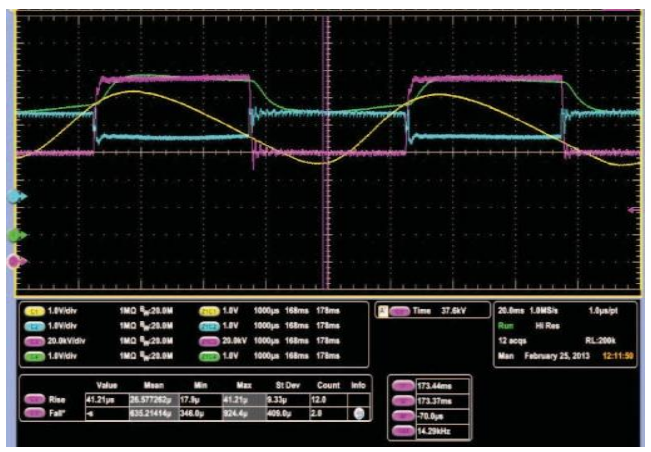

Fig 7: X-ray tube

$$
\begin{aligned}
& \text { High voltage }=-135 \mathrm{kV} \\
& \text { Low voltage }=-80 \mathrm{Kv} \\
& \text { Load current }=533 \mathrm{~mA} \\
& P P R=1500 \mathrm{~Hz} \\
& \text { Rise- and fall time }=40 \mu \mathrm{s} .
\end{aligned}
$$

The heat control by using separate hardware architecture and bit-partitioning method is illustrated in [1]. The author considered an extra efficient mechanism by inducing newer memory chips for accessing register files. Bit-partitioned Register File (BPRF) considered their designing mechanism from basic cache organization mechanism. It is designed based on a conventional dynamically scheduled superscalar processor. When test for the resistive load is started, the operation duration is from $15 \mathrm{sec}$ to $100 \mathrm{sec}$ at power of $36 \mathrm{~kW}$. All the components are also reliable. After some time, all the IGBT in the inverters have base plate start to become overheat due to which the voltage drops increase the temperature produce in such a case are $37^{\circ} \mathrm{C}$ which is the worst case.

\section{Conclusion}

In this paper a HVPS is developed which have high performance and low cost which is tested for both resistive load and also for the 
$\mathrm{X}$-ray tube. By using this technique, we are able to produce $105 \mathrm{~kW}$ to $140 \mathrm{KW}$ power at the frequency rate of $1.5 \mathrm{kHz}$ by using single tube.

\section{REFERENCES}

[1] M. P. Musau, N. A. Odero, and C. W. Wekesa. "Asynchronous interconnection of the proposed East Africa Power Pool (EAPP)," 2017 IEEE PES Power Africa, June 2017, pp. 7-11.

[2] C. Chen, K. Zhang, K. Yuan, Z. Gao, X. Teng, and Q. Ding. "Disturbance rejection-based LFC for multi-area parallel interconnected AC/DC system," IET Generation, Transmission \& Distribution, vol. 10, No. 16, pp. 4105-4117, Dec. 2016

[3] X. Zheng, Y. Chen, H. Chen, Y. Zhang, J. Zhu, J. Chen, and P. Xuan. "Loss-Minimizing Generation Unit and Tie-Line Scheduling for Asynchronous Interconnection," IEEE Journal of Emerging and Selected Topics in Power Electronics, to be published, DOI: 10.1109/JESTPE.2017.2783930

[5] H. Khan, M. U. Hashmi, Z. Khan, and R. Ahmad, "Offline Earliest Deadline first Scheduling based Technique for Optimization of Energy using STORM in Homogeneous Multi- core Systems," IJCSNS Int. J. Comput. Sci. Netw. Secur. VOL.18 No.12, December 2018, vol. 18, no. 12, pp. 125-130, 2018.

[6] H.E. Daniels et al., "Dual Voltage X-Ray Switching System”, European Patent 052 269, 1985.

[7] B. M. Gordon et al., "Dual Energy Power Supply”, US Patent 5,661,774, Aug. 26, 1997.

[8] H. Khan, M. U. Hashmi, Z. Khan, R. Ahmad, and A. Saleem, "Performance Evaluation for Secure DES-Algorithm Based Authentication \& Counter Measures for Internet Mobile Host Protocol," IJCSNS Int. J. Comput. Sci. Netw. Secur. VOL.18 No.12, December 2018, vol. 18, no. 12, pp. 181-185, 2018

[9] C. Loef and G. Zeitler, "Power Supply for an X-Ray Generator System Comprising Cascade of Two Voltage Sources", European Patent EP 2207405 B1, 2013.

[10] A. Pokryvailo, C. Carp, and C. Scapellati, "A High-Power High Voltage Power Supply for Long-Pulse Applications", IEEE Transactions on Plasma Science, Vol. 38, No. 10, October 2010, pp. 2604-2610.
[11] A. Pokryvailo, C. Carp, and C. Scapellati, "High Power, High Performance, Low Cost Capacitor Charger Concept and Implementation", IEEE Transactions on Plasma Science, Vol. 38, No. 10, October 2010, pp. 2734-2745.

[12] A. Pokryvailo, C. Carp and C. Scapellati, "Highly Efficient Switch- Mode 100-kV, 100kW Power Supply for ESP Applications", Proc. 11th Int. Conf. on Electrostatic Precipitation, Hangzhou, 21-24 Oct., 2008, pp. $284-288$ 